\title{
Article \\ On the Localized Surface Plasmonic Resonances of AgPd Alloy Nanoparticles by Experiment and Theory
}

\author{
Dimitrios Ntemogiannis ${ }^{1}$, Maria Tsarmpopoulou ${ }^{1}$, Alexandros G. Chronis ${ }^{1} \oplus$, Dimitrios I. Anyfantis ${ }^{1} \oplus$, \\ Alexandros Barnasas ${ }^{1}\left(\mathbb{D}\right.$, Spyridon Grammatikopoulos ${ }^{2}\left(\mathbb{0}\right.$, Mihail Sigalas ${ }^{1}$ and Panagiotis Poulopoulos ${ }^{1, *(1)}$ \\ 1 Materials Science Department, University of Patras, 26504 Patras, Greece; \\ dim.ntemogiannis@gmail.com (D.N.); mariatsarmpopoulou@gmail.com (M.T.); \\ a.chronis@upatras.gr (A.G.C.); up1057157@upatras.gr (D.I.A.); mparnalex@gmail.com (A.B.); \\ sigalas@upatras.gr (M.S.) \\ 2 Department of Mechanical Engineering, University of Peloponnese, M. Alexandrou 1, 26334 Patras, Greece; \\ spiridongramma@upatras.gr \\ * Correspondence: poulop@upatras.gr; Tel.: +30-2610-997501
}

\section{check for}

updates

Citation: Ntemogiannis, D. Tsarmpopoulou, M.; Chronis, A.G.; Anyfantis, D.I.; Barnasas, A.; Grammatikopoulos, S.; Sigalas, M.; Poulopoulos, P. On the Localized Surface Plasmonic Resonances of AgPd Alloy Nanoparticles by Experiment and Theory. Coatings 2021, 11, 893. https://doi.org/ 10.3390/coatings 11080893

Academic Editor: Torsten Brezesinski

Received: 1 July 2021

Accepted: 23 July 2021

Published: 26 July 2021

Publisher's Note: MDPI stays neutral with regard to jurisdictional claims in published maps and institutional affiliations.

Copyright: (C) 2021 by the authors. Licensee MDPI, Basel, Switzerland. This article is an open access article distributed under the terms and conditions of the Creative Commons Attribution (CC BY) license (https:// creativecommons.org/licenses/by/ $4.0 /$ )

\begin{abstract}
Ag} / \mathrm{Pd}$ multilayers and $\mathrm{AgPd}$ alloyed ultrathin films were deposited on Corning glass by magnetron sputtering. After being annealed in a furnace in air at $460{ }^{\circ} \mathrm{C}$, self-assembled nanoparticles were formed. Localized surface plasmon resonances were observed only for the Ag-rich samples in the full range of the visible light spectrum. The resonance position was found to depend on the initial film thickness. In order to gain further physical insight, rigorous theoretical calculations were carried out via the rigid coupled-wave analysis method for the entire compositional range between $\mathrm{Ag}$ and Pd. Theoretical calculations were proven to be in suitable agreement with the experimental results.
\end{abstract}

Keywords: growth; silver; palladium; nanoparticles; optical properties; plasmons; RCWA

\section{Introduction}

Noble metal NanoParticles (NPs) with diameters in the nanoscale range show remarkable properties with respect to the photon absorption phenomenon. The appearance of Localized Surface Plasmon Resonances (LSPRs) makes these materials suitable for a great variety of applications nowadays. In particular, LSPRs are being used in Scanning NearField Optical Microscopy (SNOM), in Photon Scanning Tunneling Microscopy and for light trapping in photovoltaic systems. Furthermore, LSPRs have been assayed in nanosensors, gas sensors and biosensors, for the detection of humidity and gases in the environment, for the surface-enhanced of Raman scattering, for field enhanced spectroscopies, to the non-linear effects; see, for example, Refs. [1-9] and references therein.

So far, numerous works delivered on LSPRs are basically oriented toward Ag and Au NPs. However, the continuous research for novel materials with special properties in the areas of hydrogen and photocatalysis has emerged new metals, such as Pt and Pd, on the screenplay [10-14]. In this study, we work with AgPd plasmonic NPs. AgPd NPs were produced by initially depositing $\mathrm{Ag} / \mathrm{Pd}$ multilayer structures and then annealing them in air. By tuning the thickness of the layers, we could change the composition of the NPs. We determined the optimum composition for obtaining LSPRs. Then, we showed that, alternatively, if using as starting material co-deposited $\mathrm{AgPd}$ alloy films of the optimum composition, we could reproduce the LSPRs. In all cases examined, the annealed films show broad LSPRs due to the presence of AgPd NPs. Thinner films result in smaller NPs and LSPRs located at about $2.7 \mathrm{eV}$, while thicker films result in larger NPs showing LSPRs with some considerable red shift. The combination of both thicker and thinner films covers the full range of optical light by LSPRs. Finally, the experimental results are discussed with respect to theoretical calculations performed with the help of the Rigorous Coupled-Wave Analysis (RCWA) in order to obtain a deeper physical insight $[15,16]$. 
We have to notice here that the most straightforward method to fabricate AgPd nanoparticles would be to perform annealing under vacuum or in a furnace filled with an inert gas [17,18]. Another more elaborate but also more costly method would be to employ nanolithography [19]. However, the most cost-effective method would be to perform the annealing in air. The limits of this method are tested here. Silver (Ag) and Palladium (Pd) show opposite behavior concerning oxidation in air: the annealing of silver oxides in air at temperatures higher than $150{ }^{\circ} \mathrm{C}$ results in the decomposition of them [20]. Therefore, $\mathrm{Ag}$ in air atmosphere at $460{ }^{\circ} \mathrm{C}$ is metallic. On the other hand, Pd gets gradually oxidized at temperatures above $300{ }^{\circ} \mathrm{C}$ approximately [21]. Since temperatures needed for obtaining self-organized NPs in air are higher than about $350{ }^{\circ} \mathrm{C}[22,23]$, it is important to keep oxidation of $\mathrm{Pd}$ at a minimal level. $\mathrm{PdO}$ is a semiconductor with an optical bandgap at about $2.2 \mathrm{eV}$ [24]. The presence of a small discontinuity in the absorbance spectra at $2.2 \mathrm{eV}$ could then be the fingerprint for the detection of PdO. Similarly, in $\mathrm{Ag}(\mathrm{NiO}) \mathrm{com}-$ posite materials, we could always detect the bandgap edge of $\mathrm{NiO}$ [25].

\section{Materials and Methods}

\subsection{Experimental Details}

Before ending up with a suitable AgPd alloy NP system, which would not show Pd oxidation effects, we tried to evaluate the composition along with the sequence of the deposited thin Ag/Pd multilayers on Corning glass. By tuning the thickness of the individual Ag and Pd layers, one may actually tune the composition.

For this purpose, multilayers were deposited by radio frequency magnetron sputtering under high vacuum conditions (base pressure $\sim 5 \times 10^{-7} \mathrm{mbar}$ ). Ag and Pd targets were put on two sputtering heads (the Torus $2 \mathrm{HV}$ circular sputtering source of Kurt J. Lesker Company, Jefferson Hills, PA, USA). In order to determine the deposition rates of Ag and $\mathrm{Pd}$ and calibrate the quartz balance thickness monitor (Inficon XTM/2, accuracy $\pm 0.1 \mathrm{~nm}$, bought also from Kurt J. Lesker), we firstly prepared an $80 \mathrm{~nm}$ thick film composed of Pd buffer and coating layers and an $\mathrm{Ag} / \mathrm{Pd}$ multilayer between them. $\mathrm{Ag}$ and $\mathrm{Pd}$ are fully miscible, and if one runs high-angle x-ray diffraction (XRD), the pattern consists of a solid solution (average composition) peak surrounded by satellites. By using the XRD pattern (recorded with a Bruker, D8-Advance, Karlsruhe, Germany) and the Vegard equation, one may determine the composition and the thickness of the Ag and Pd layers [26]. Deposition rates were found to be $0.12 \mathrm{~nm} / \mathrm{s}$. Via in-house software analysis [26], the full XRD pattern can be fitted, Figure 1. The full miscibility of the elements, on the other hand, guarantees that after annealing at $460{ }^{\circ} \mathrm{C}$, the multilayer will turn into an alloy by interdiffusion $[27,28]$.

After evaluating the proper composition for LSPRs to be observed, for comparison reasons, we modified the deposition method by employing a cost-effective medium vacuum chamber with our sputter coater device (modified Balzers Union model SCD040, Oerlikon Balzers, Balzers, Liechtenstein) [23]. We directly fabricated the desired AgPd alloy film by co-deposition of $\mathrm{Ag}$ and $\mathrm{Pd}$, implementing the same method applied for fabricating $\mathrm{Cu}$ and $\mathrm{Au}$ alloy films in Ref. [29]. A Pd target was placed under an Ag target. On the Ag target, we drilled the appropriate number of holes so that the concentration of $\mathrm{Pd}$ in the alloy is the desired one.

The morphology of AgPd films after annealing was probed by Atomic Force Microscopy (AFM) performed with the help of a Multimode Microscope with a Nanoscope IIIa controller and a $120 \times 120 \mu \mathrm{m}^{2}$ magnet-free scanner (Model AS-130VMF) developed by Digital Instruments (Chapel Hill, NC, USA). The microscope operated in the non-contact (tapping) mode [30].

Finally, the UV-Vis spectra were recorded at room temperature in the transmission geometry with the help of a Shimadzu UV-Vis Spectrophotometer, Model: UV 1800 (Shimadzu, Kyoto, Japan) at wavelengths 200-1100 nm. 


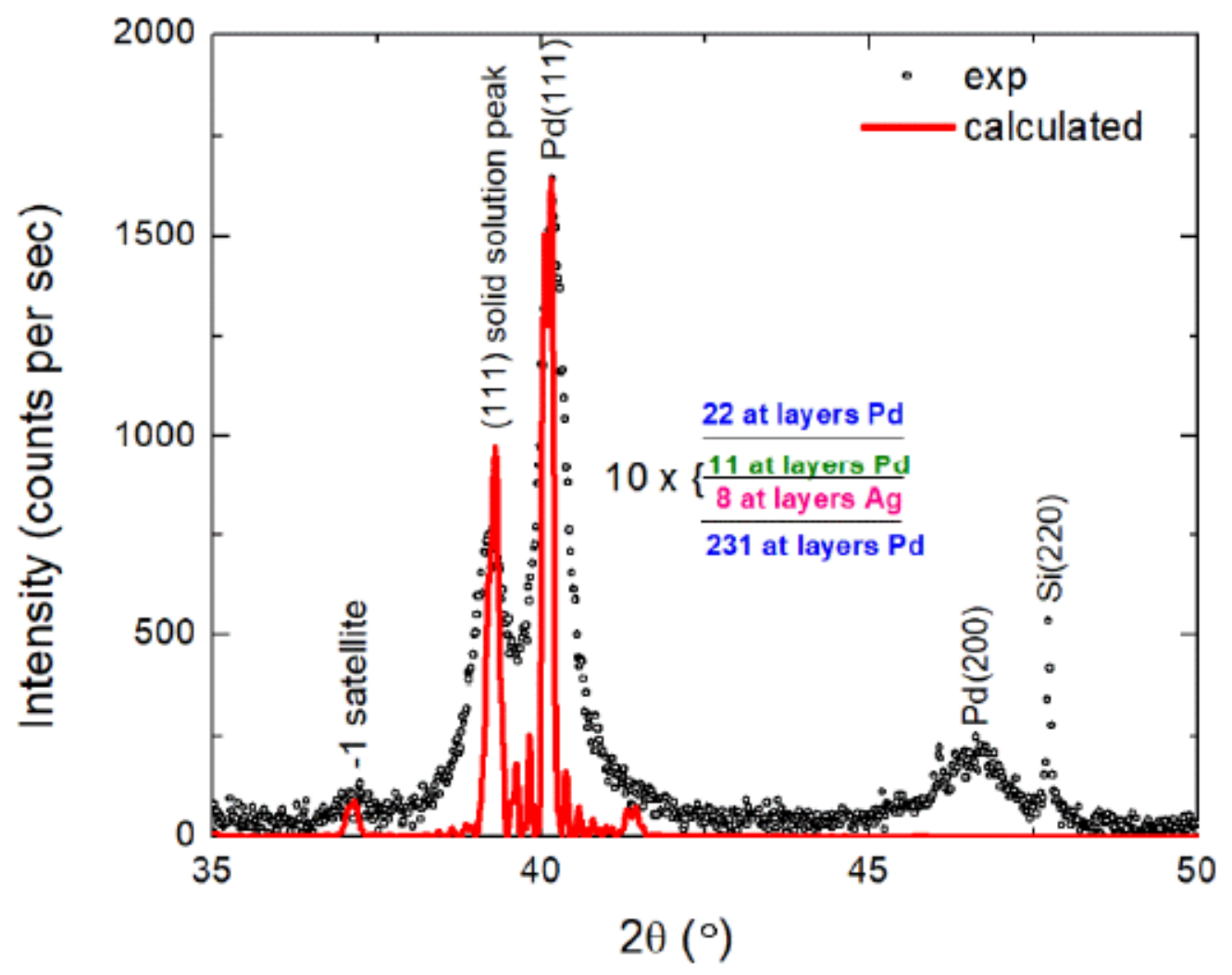

Figure 1. Experimental and calculated high-angle XRD pattern of an $\mathrm{Ag} / \mathrm{Pd}$ multilayer grown over a relatively thick Pd buffer layer and capped by a thin Pd layer. This sample was grown on a Si (100) wafer to minimize the noise in the XRD pattern. The Pd diffraction served as a ruler for the accurate determination of the angular position of the diffraction peak of the multilayer. This, together with the position of the first-order satellite diffraction were necessary for the accurate (within 5\%) determination of the Pd concentration and the thickness of the individual Ag and Pd layers in the number of atomic (at) layers as indicated [26].

\subsection{Theoretical Model}

Rigorous Coupled-Wave Analysis (RCWA), an analytical approach for interpreting the diffraction of an electromagnetic plane wave incident bounded by two media [15], is a fast computational method, using the Fourier series in order to distinguish the investigated areas into layers, making all calculations easier. The RCWA theory is applied for cubes and cylinders, having the same cross-section along the $z$-axis.

Within each layer, the relative permittivity has a two-dimensional periodicity and can be expanded in the following Fourier series.

$$
\varepsilon(x, y, z)=\varepsilon(x+\Lambda, y+\Lambda, z)=\sum_{p, q} \varepsilon_{p q}(z) e^{i\left(p K_{x} x+q K_{y} y\right)}
$$

where $\Lambda$ is the grating period, $\varepsilon_{p q}$ the Fourier component of grating permittivity $K_{x}=K_{y}=2 \pi / \Lambda$ and $i=(-1)^{1 / 2}$

The electric field of the incoming region can be described with the next formula.

$$
\mathrm{E}_{\mathrm{I}}=\mathrm{e}^{-\mathrm{i}\left(k_{x i} x+k_{y i} y+k_{z i} z\right)}+\sum_{\mathrm{nm}} R_{n m} \mathrm{e}^{-\mathrm{i}\left(k_{x n} x+k_{y m} y+k_{z n m i} z\right)}
$$

where $k_{x n}=k_{x 0}+n K_{x}, k_{y n}=k_{y 0}+m K_{y}, k_{z n m i}=\sqrt{ }\left(k^{2} \varepsilon_{i}-k^{2} x n-k_{y m}^{2}\right), k_{x 0}$ and $k_{y 0}$ are the $x$ and $y$ components of the incident plane wave, and $k=2 \pi / \lambda, R_{n m}$ is the $n, m$ order backward diffracted wave. 
The electric field in each layer is:

$$
E_{I I}=\sum_{n m} S_{n m}(z) e^{-i\left(k_{x n} x+k_{y m} y+k_{z n m i} z\right)}
$$

where $S_{n m}(z)$ are the space harmonics field amplitudes.

The electric field in the outgoing region is:

$$
E_{I I I}=\sum_{n m} T_{n m} e^{-i\left(k_{x n} x+k_{y m} y+k_{z n m o}(z-d)\right)}
$$

where $k_{z n m o}=\sqrt{ }\left(k_{\varepsilon o}^{2}-k_{x n}^{2}-k_{y m}^{2}\right), T_{n m}$ is the $n, m$ order forward diffracted wave.

Considering $n=\mathrm{N}$ and $m=\mathrm{M}$ while keeping the continuity of the tangential electric and magnetic fields at $z=0$ and $z=d$ boundaries, then a $4 \times 4$ system of four equations and four unknown emerges, resulting in the solution of $R_{n m}$ and $T_{n m}$ values.

As RCWA requires less computational time, it outperforms other solution methods such as Density Functional Theory (DFT) and Time-Dependent Density Functional Theory (TD-DFT), which need to increase their mesh linearly as this length increases, reducing that way the computational time [25].

The dielectric function of the metals $(\mathrm{Ag}, \mathrm{Pd})$ was directly calculated from the study of Rakic et al. about the optical properties of metallic films [31]. To calculate the dielectric constant of the alloy, the dielectric of each metal is added and is multiplied with the corresponding percentage. The refractive index of the $\mathrm{SiO}_{2}$ was 1.45.

\section{Results}

\subsection{Uv-Vis Spectra of AgPd Nanoparticles}

UV-vis absorbance spectra plotted as a function of photon energy for two initially layered structures of $\mathrm{Pd}$ and $\mathrm{Ag}$, with thicknesses as indicated, are illustrated in Figure 2. Films have been annealed at $460^{\circ} \mathrm{C}$ in air. For the bilayer of Figure 2a, no LSPR is observed. On the contrary, the graph witnesses the presence of a PdO absorption edge. This becomes evident from the inset of Figure $2 \mathrm{a}$, where is presented the absorbance spectrum of a mere PdO thin film. This absorption edge looks similar to one of the direct bandgap semiconductors, $\mathrm{NiO}$ [32] or $\mathrm{ZnO}$ [33]. In Figure 2b, the corresponding spectra for an initial four-layer film after annealing are plotted. The initial individual layer thicknesses are indicated in the inset drawings. Once again, the PdO edge can be observed; however, some broad LSPRs can also be identified. Figure 2 demonstrates that if the layered structures finish in $\mathrm{Pd}$ and/or the Pd is of significant concentration. The oxidation process is faster than the interdiffusion resulting in samples with no interesting features for plasmonics. This leads us to seek for LSPRs only in Ag-rich alloyed NPs.

The desired results came only after we decreased the Pd concentration to about 15 at. $\%$. This is depicted in the absorbance spectra of Figure 3 for two initially layered structures (the thickness is indicated) after being annealed at $460{ }^{\circ} \mathrm{C}$ in air. Spectra show only LSPRs and no sign of PdO. The thinner sample (trilayer) after annealing shows LSPRs with a peak close to the one of Ag NPs [23]. The thicker sample (five-layer) displays LSPRs red-shifted compared to the ones of the trilayer. This may be interpreted after comparing these results to the ones of Ref. [23], where thicker Ag films after annealing resulted in larger NPs with red-shifted resonances. Two principal outcomes are stated: Firstly, the LSPRs are very broad compared to those of Ag NPs, see, e.g., Ref. [23]. However, this is not surprising if compared to the few literature data existing for AgPd NPs [10-14]. In the latter references, most of the LSPRs are broad and located near the red edge of the visible spectrum. As analyzed in the next paragraphs, this broadness may be attributed to the broad LSPRs calculated for net Pd NPs. Secondly, the LSPRs of the two samples together cover the entire range of the visible spectrum; therefore, they can be potentially appropriate for any LSPR application, especially photocatalysis. 

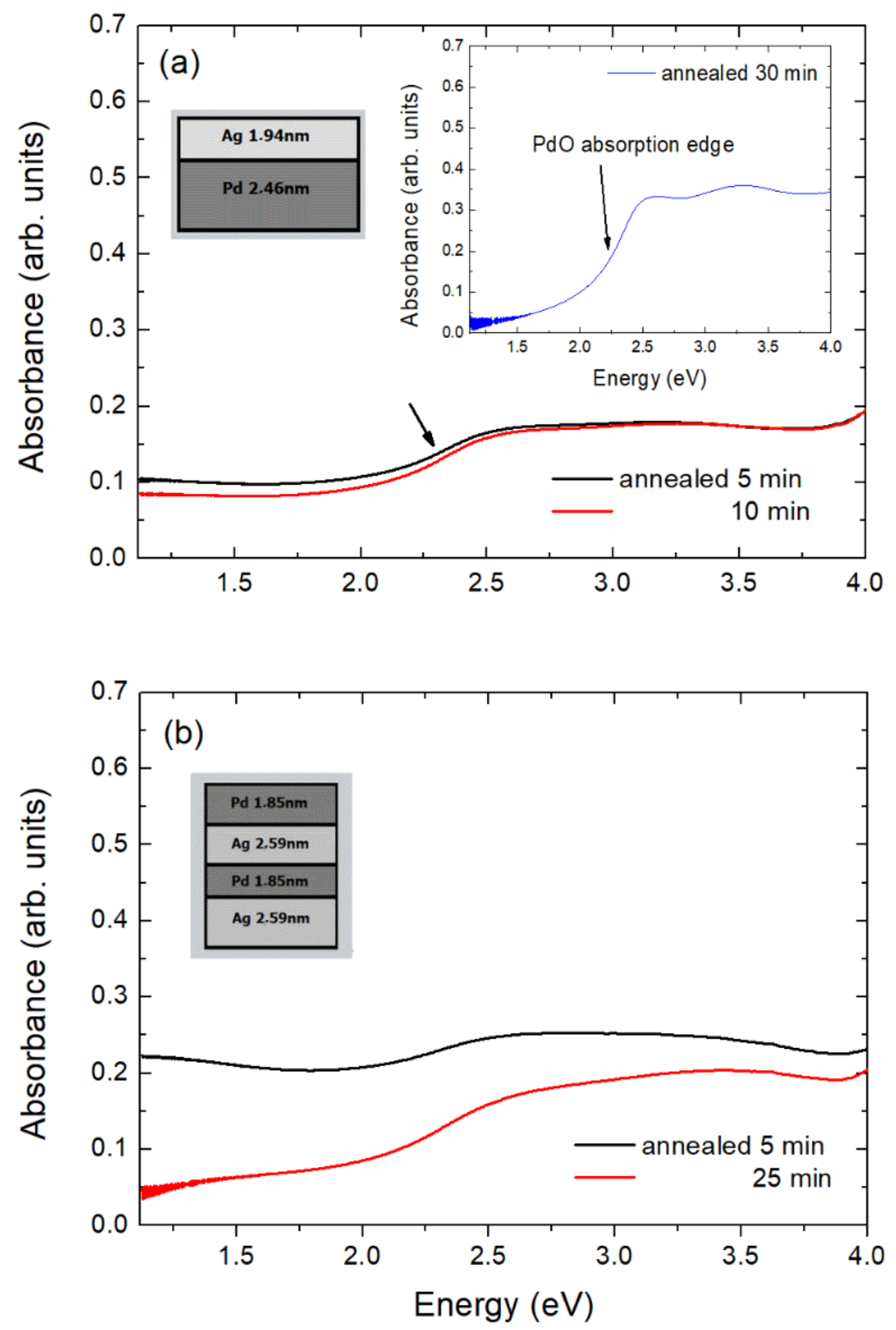

Figure 2. Absorbance spectra for thin $\mathrm{Ag} / \mathrm{Pd}$ layered films grown on Corning glass and annealed at $460{ }^{\circ} \mathrm{C}$. The relatively large concentration of $\mathrm{Pd}$ resulted in the formation of $\mathrm{PdO}$ (the edge is marked with arrows). Practically no LSPRs were detected in (a), while in (b), some broad LSPRs appear. For comparison, the $\mathrm{PdO}$ absorption edge recorded on a $10 \mathrm{~nm}$ film is plotted as an inset in (a). 


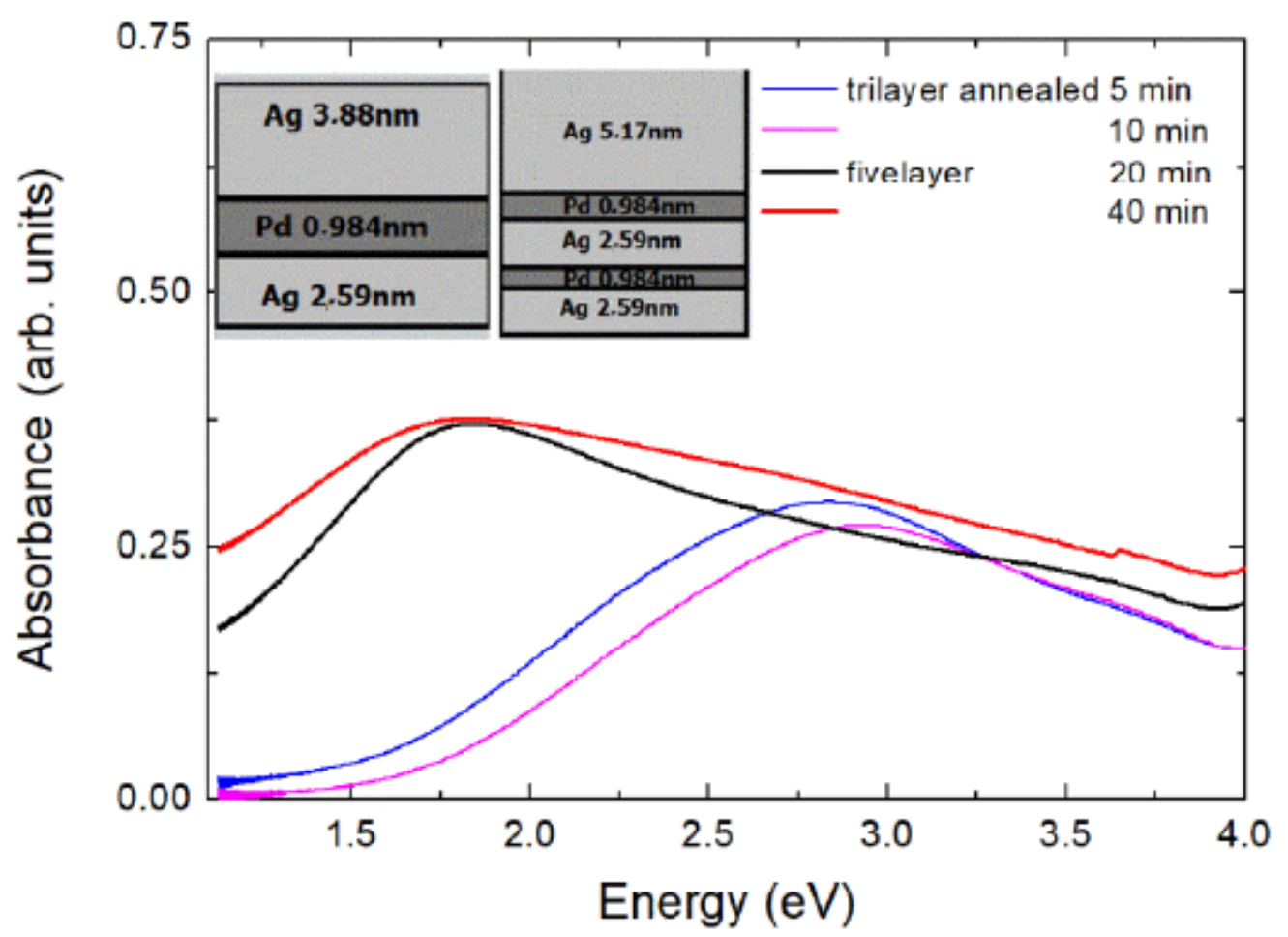

Figure 3. Absorbance spectra for thin $\mathrm{Ag} / \mathrm{Pd}$ layered films on Corning glass annealed at $460{ }^{\circ} \mathrm{C}$ in air. One may see broad LSPRs with a maximum at about the position of Ag NPs one, compared to Ref. [23]. It seems that the thick Ag layers on the top protected the film against oxidation and the very thin Pd ones had the time to inter-diffuse completely with the Ag ones in order for the AgPd NPs to be formed, resulting in the appearance of LSPRs.

In Figure 4a, we indicatively present the AFM image of the surface of the initial trilayer film of Figure 3 after annealing. The film has been transformed into self-assembled NPs, relatively homogeneous with respect to the NPs size. Nanoparticle-diameter size distribution follows well a Gaussian line, and it is exhibited in Figure $4 \mathrm{~b}$. The NPs have a mean diameter slightly larger than $100 \mathrm{~nm}$. The NPs height is about 30-50 nm. The main reason we did this AFM experiment was to prove the presence of NPs and, additionally, to support the theoretical calculations with real geometrical parameters.

For completeness's sake, in Figure 5, we present UV-Vis spectra for films of the same initial thickness and concentration as described above, directly produced as alloys via the co-deposition method. The alloy films were successively annealed under the same experimental conditions as the films in Figure 3. One may notice that the basic LSPR features of the alloyed films are similar to the corresponding ones of the layered films for the same total film thickness plotted in Figure 3.

\subsection{Theoretical Results and Discussion}

The experimental results described in the previous paragraphs of the present study are being supported by theoretical work in the context of 3.2 paragraph. Although the behavior of Ag NPs has been thoroughly examined in Ref. [23], it is briefly presented here to further enhance the experimental outcomes on LSPRs' appearance in the UV-Vis spectrum of AgPd NPs. Attention was given to the synthesis of the bimetallic alloy of AgPd along with the changes of thickness ( $t$ ), diameter $(d)$ and lattice constant (a) values in order to better simulate the produced samples. 

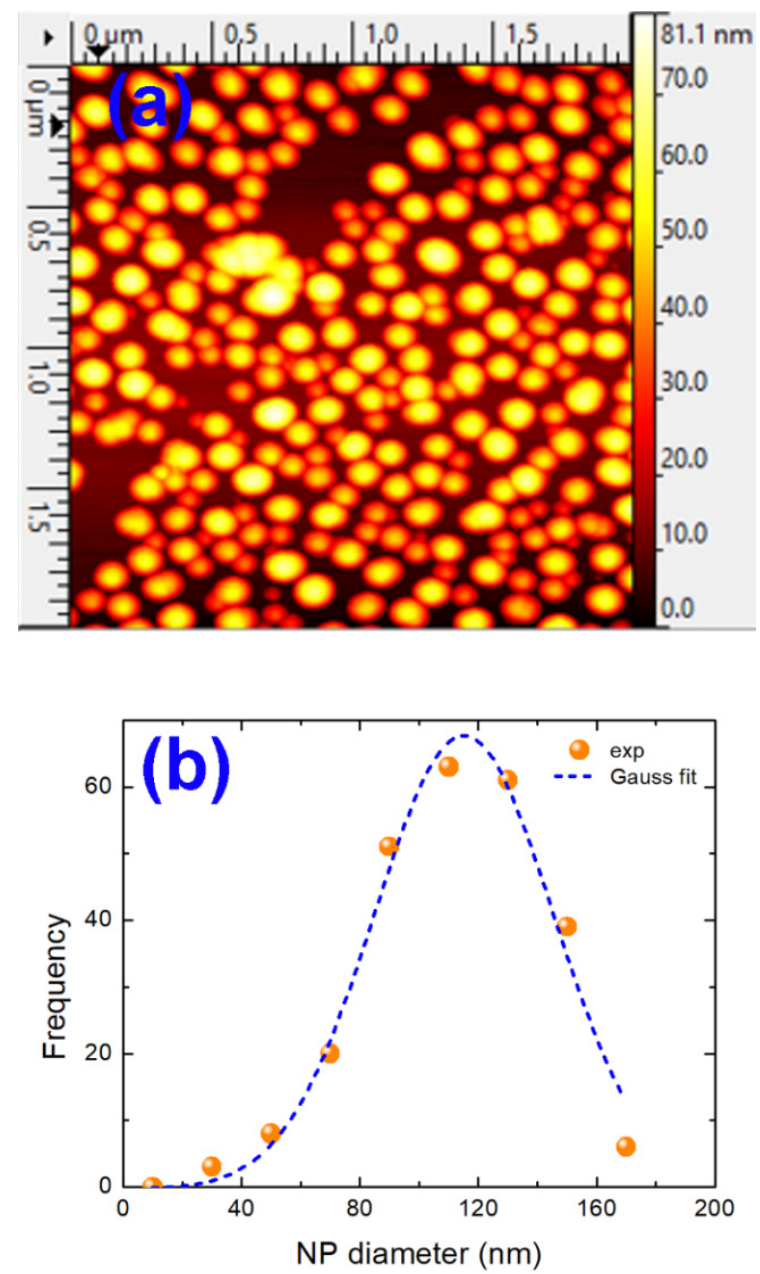

Figure 4. (a) AFM image of the surface of the annealed film of Figure 3 (trilayer). The image shows NPs with a diameter slightly larger than $100 \mathrm{~nm}$. (b) The relevant diameter size distribution.

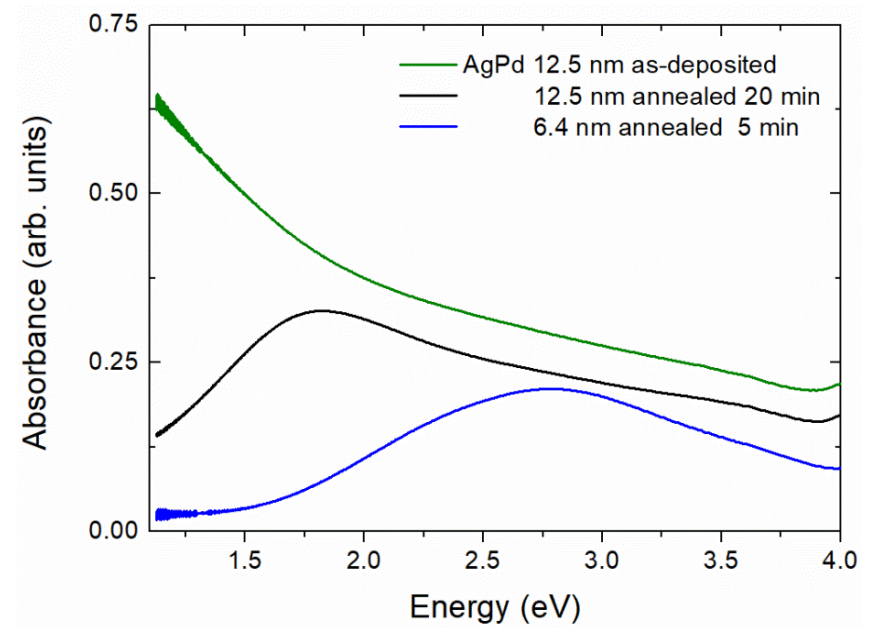

Figure 5. Absorbance spectra for thin AgPd alloyed films on Corning glass annealed at $460{ }^{\circ} \mathrm{C}$ in air. One may see broad LSPRs with maxima close to the position of Ag NPs one, compare to Ref. [20]. The results look similar to the ones in Figure 3, showing that the LSPRs shape and position depend on the total initial film thickness and Pd concentration. For comparison's sake, the spectrum for the non-annealed $12.5 \mathrm{~nm}$ thick film is presented; it shows a typical metallic behavior [23,34]. 
The majority of scenarios investigated concern bimetallic alloys of $85 \% \mathrm{Ag}$ and $15 \%$ Pd. Extra computational time was allocated for the cases of 50\% Ag and 50\% Pd, 100\% $\mathrm{Ag}$ and $100 \% \mathrm{Pd}$. The values of the geometry parameters simulated were either diameter equals to $d=100 \mathrm{~nm}$ or $d=200 \mathrm{~nm}$; lattice constant $\mathrm{a}=200$ or $300 \mathrm{~nm}$, along with thickness $\mathrm{t}$ ranging between $10 \mathrm{~nm}<\mathrm{t}<50 \mathrm{~nm}$.

Figure 6 illustrates the examined system of the AgPd alloy. NPs are represented as cubes and cylinders because they are effectively treated in a shorter computational time when applying the RCWA method [16]. The NPs are placed on top of $\mathrm{SiO}_{2}$. It is proven from previously published articles $[23,25]$ that despite the non-periodical behavior of material fabricated via experimental methods, there is practically a suitable agreement between the theoretical and experimental results. To calculate the disorder structure found experimentally is very difficult, if not impossible, because it needs a calculation in a big supercell, which is time and memory consuming. On the other hand, calculating one individual metal nanoparticle is not reliable because, experimentally, we see the nanoparticles to be very close to each other. For that reason, the periodic array of nanoparticles was used in the calculations. Of course, we expect the absorbance to be higher than the measurements [35].
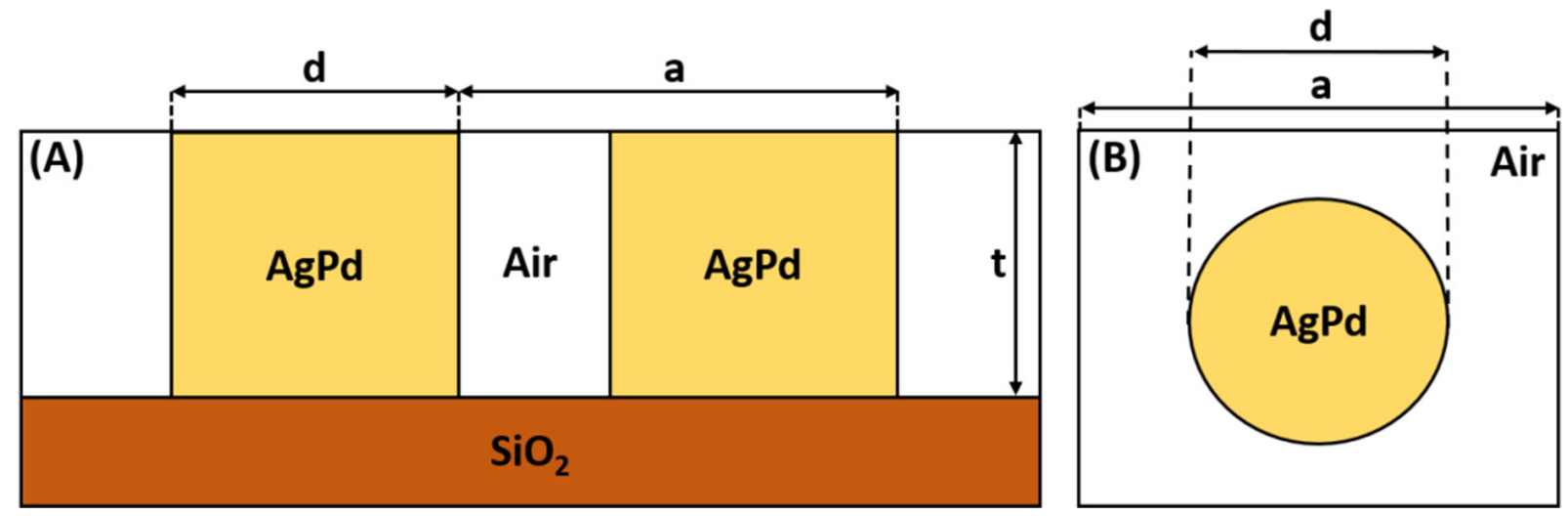

Figure 6. The examined system of AgPd alloy (side view (A) and top view (B)), where a is the lattice constant, $d$ the diameter and $\mathrm{t}$ the thickness of the nanoparticles.

It is highlighted that as the participation of Pd to the alloy is increased, the resonances obtain broader till they totally dampen from the UV-Vis spectrum. Only when the sample fully comprises Ag, two LSPRs show up, both well defined. Peaks appearing in the UV-Vis spectrum of the pure Ag case ran are due to the presence of the $\mathrm{SiO}_{2}$ substrate [23]. When removing the $\mathrm{SiO}_{2}$ substrate, the two peaks are smoothened. Similar behavior is observed for the Ag-rich AgPd alloy in Figure 7.

Figure 7 exhibits the decrease in Ag contribution in the alloy toward the appearance of LSPRs. The diameter of NPs equals to $d=100 \mathrm{~nm}, \mathrm{a}=200 \mathrm{~nm}$ and $\mathrm{t}=50 \mathrm{~nm}$. Starting from 100 at.\% Ag presence (blue graph), gradually reducing to 85 at.\% Ag and 15 at.\%. Pd (red graph), to 50 at.\% $\mathrm{Ag}$ and 50 at.\% Pd alloy synthesis (green graph), further reducing the Ag concentration to 25 at.\% Ag and 75 at.\% Pd (dark yellow graph) we end up with 100 at.\% Pd synthesis (black graph). Desired results proving the existence of LSPRs in accordance with the experimental ones are fairly reproduced with the decrease in Pd concentration in the alloy.

Case of 50 at.\% Ag and 50 at.\% Pd (green graph of Figure 7) is well compared to the graph of Figure $2 \mathrm{~b}$ due to the alike composition of the AgPd film. LSPRs broadness is more evident when it comes to the 85 at.\% $\mathrm{Ag}$ and 15 at.\% Pd alloy, hence is more analyzed below. Unlike the composition of the alloy, which is kept stable, several scenarios were investigated in terms of the NPs size. 


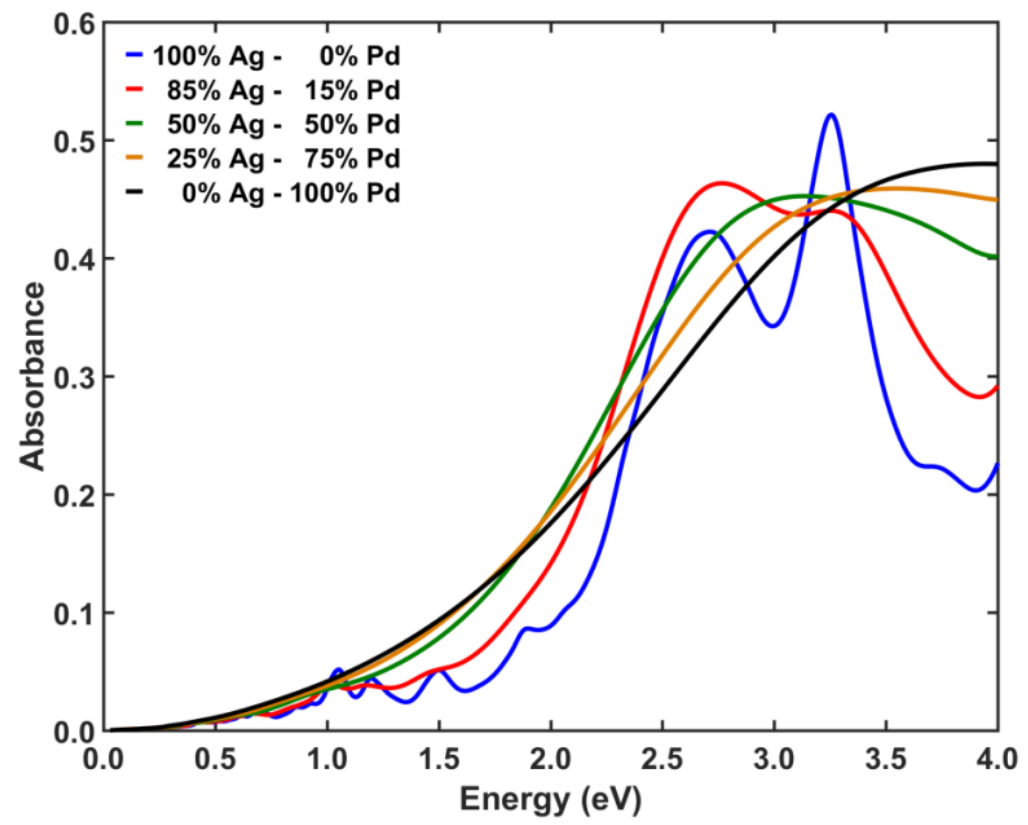

Figure 7. Different syntheses of the AgPd alloy reflecting the existence of LSPRs. The parameters are: $\mathrm{d}=100 \mathrm{~nm}, \mathrm{a}=200 \mathrm{~nm}, \mathrm{t}=50 \mathrm{~nm}$.

In Figure 8, the dependence of the plasmonic resonances against thickness is presented when diameter remains constant $d=100 \mathrm{~nm}$ and lattice constant $\mathrm{a}=200 \mathrm{~nm}$. Thickness varies from $t=30$ to $50 \mathrm{~nm}$, like in the experiment. The increase in absorbance is monitored with the increase in $t$ while there is a short blue shift of the resonances to the UV spectrum. One main LSPR is observed at $2.46 \mathrm{eV}$ when $\mathrm{t}=30 \mathrm{~nm}$ and at $2.6 \mathrm{eV}$ when $\mathrm{t}=40 \mathrm{~nm}$ and two (2) broad resonances at 2.76 and $3.29 \mathrm{eV}$, respectively for $\mathrm{t}=50 \mathrm{~nm}$. Curves are compared to those of Figure 3 (trilayer) as the mean diameter of NPs is measured close to $100 \mathrm{~nm}$ according to the AFM images along with the synthesis of NPs, which consist of about 84 at.\% Ag and 16 at.\% Pd.

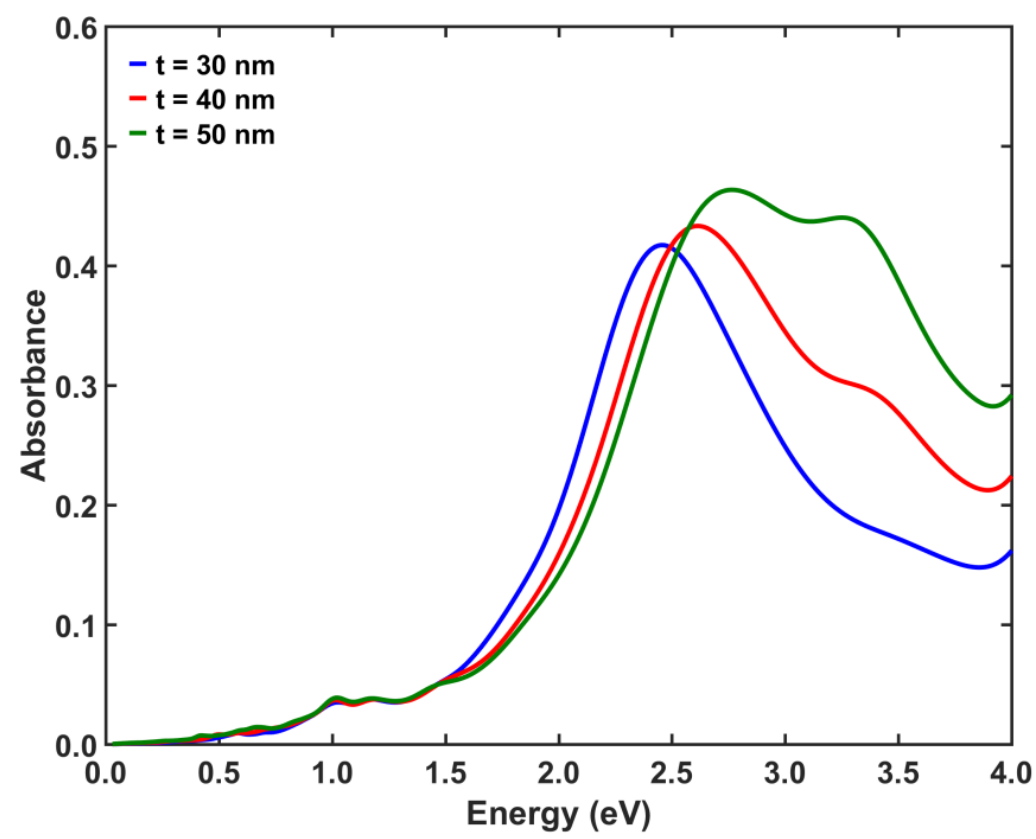

Figure 8. The absorbance spectrum of 85 at. $\% A g$ and 15 at. $\% \mathrm{Pd}$, when $\mathrm{d}=100 \mathrm{~nm}, \mathrm{a}=200 \mathrm{~nm}$. 
Extra simulations were also performed, keeping the synthesis of NPs the same as previously but changing the diameter to $d=200 \mathrm{~nm}$ and lattice constant $\mathrm{a}=300 \mathrm{~nm}$, see Figure 9. Thickness values were set $t=30$ and $40 \mathrm{~nm}$. Resonances are still distinguished for the thicker NPs of $d=200 \mathrm{~nm}$ compared to the resonances of the thinner films of $\mathrm{d}=100 \mathrm{~nm}$. Blue shift for increased thickness is also observed. Maximum absorbance is measured at $1.83 \mathrm{eV}$ for $\mathrm{t}=30 \mathrm{~nm}$ and $2.0 \mathrm{eV}$ for $\mathrm{t}=40 \mathrm{~nm}$, nicely fitted to the experimental results of Figure 3 (five-layer).

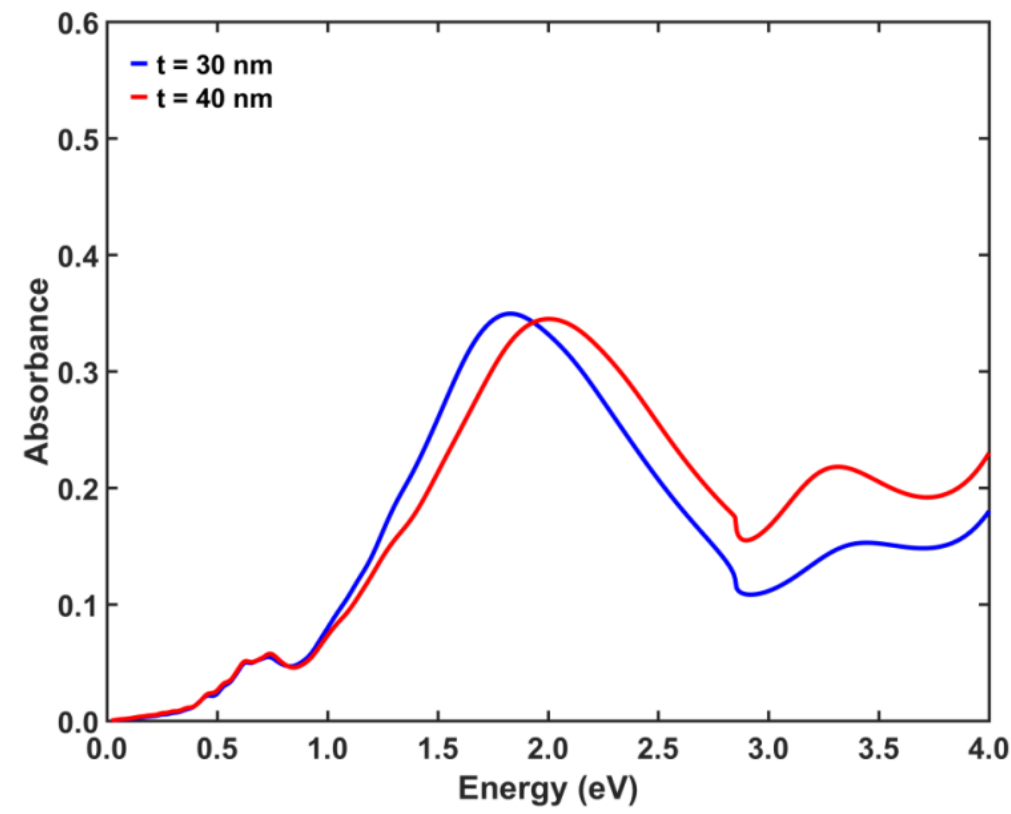

Figure 9. The absorbance spectrum of 85 at.\%Ag and 15 at. $\%$ Pd, when $d=200 \mathrm{~nm}, \mathrm{a}=300 \mathrm{~nm}$.

The dependence of LSPRs was further evaluated in terms of thickness $t$, particularly examining the 85 at.\% $\mathrm{Ag}$ and 15 at.\% Pd alloy of $\mathrm{d}=100 \mathrm{~nm}$ and $\mathrm{a}=200 \mathrm{~nm}$, along with $\mathrm{t}=30 \mathrm{~nm}$ (blue curve of Figure 8), but decreasing the $\mathrm{t}$ value from 30 to 15 and $10 \mathrm{~nm}$, respectively. It is obvious that the decrease in the $t$ value causes the red shift of the resonance and a slight drop of the absorbance max value, respectively. Both graphs for either $\mathrm{t}=10 \mathrm{~nm}$ or $\mathrm{t}=15 \mathrm{~nm}$ reflect steeper curves compared to the curve of Figure 8 (blue graph), supporting the experimental results that the shape and position of LSPRs are straightforward to the thickness of AgPd NPs. Figure 10 illustrates the absorbance spectra with the reduced values of thickness. The blue graph depicts the absorbance spectrum when $t=10 \mathrm{~nm}$, and the red graph depicts the spectrum when $t=15 \mathrm{~nm}$.

From the comparative work implemented via software analysis and experiment, the graphs illustrated in Figure 3 (experiment) and Figures 8 and 9 (software) show remarkable similarities in terms of LSPR features. For the three-layer sample (blue curve-Figure 3) it presents a single resonance at $2.8 \mathrm{eV}$ and maximum absorbance at 0.32 absorbance units (a.u.), whereas the max absorbance for the NPs simulated of $\mathrm{d}=100 \mathrm{~nm}$ and $\mathrm{a}=200 \mathrm{~nm}$ (Figure 8) also present a single resonance at 2.46, 2.6 and $2.76 \mathrm{eV}$, close to the LSPR position of the three-layer. The range of values is due to the increased thickness of the NPS for $\mathrm{t}=30,40$ and $50 \mathrm{~nm}$. It is worth mentioning that the mean diameter of the three-layer NPs measured according to the AFM images is slightly bigger than $100 \mathrm{~nm}$. As well, the five-layer (black graph-Figure 3) resembles the behavior of the thicker NPs simulated of $\mathrm{d}=200 \mathrm{~nm}$ and $\mathrm{a}=300 \mathrm{~nm}$ (Figure 9). The latest exhibit resonance at 1.83 and $2 \mathrm{eV}$ with the increasing thickness from $\mathrm{t}=30$ to $40 \mathrm{~nm}$, while the five-layer shows LSPR at $1.8 \mathrm{eV}$. Maximum absorbance values for both the examined spectra of UV-Vis located at 0.35 a.u. 


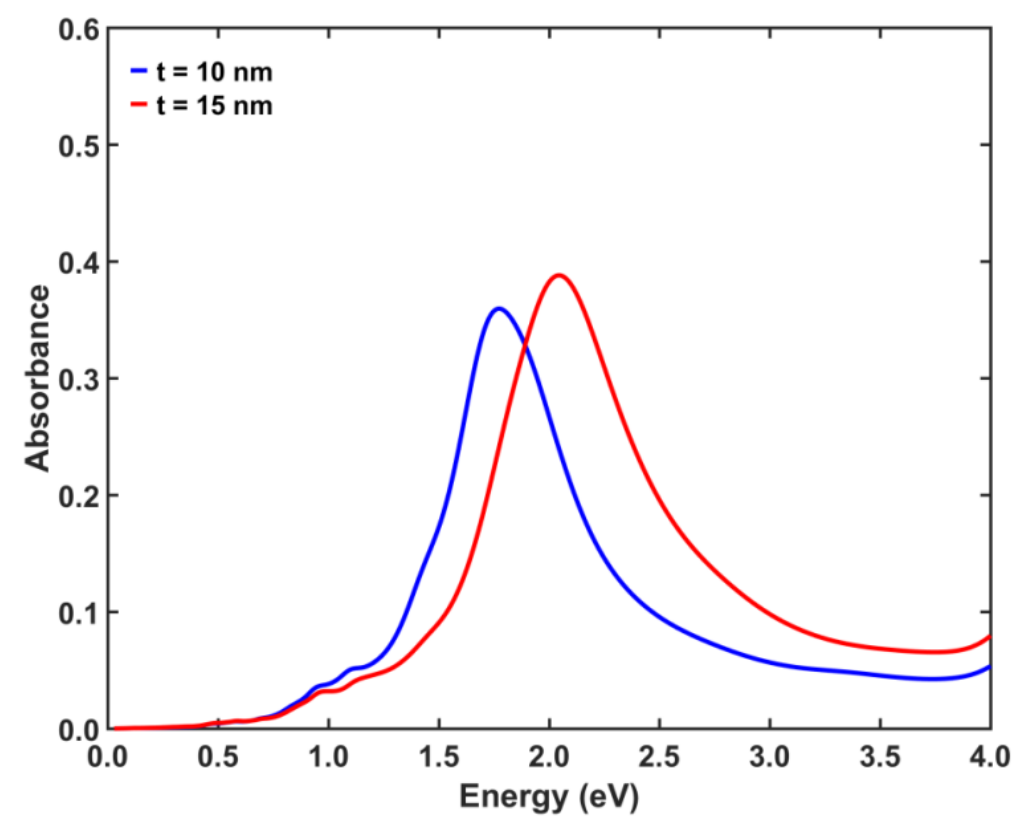

Figure 10. The absorbance spectrum of 85 at.\% $\mathrm{Ag}$ and 15 at.\%Pd, with reduced $\mathrm{t}$ values, when $\mathrm{d}=100 \mathrm{~nm}$ and $\mathrm{a}=200 \mathrm{~nm}$.

\section{Conclusions}

In this work, we fabricated AgPd nanoparticles showing LSPRs after annealing at $460{ }^{\circ} \mathrm{C}$ in air initially layered $\mathrm{Ag} / \mathrm{Pd}$ films or $\mathrm{AgPd}$ alloyed thin films thinner than $20 \mathrm{~nm}$. Only Ag-rich films delivered the desired results. The combination of both thicker and thinner films covers the full range of optical light by LSPRs, making this material potentially interesting for hydrogen storage applications and photocatalysis. RCWA theory correctly predicted the position and the LSPRs features depending on the nanoparticle size. Conclusively, the augmented presence of $\mathrm{Pd}$ results in no resonances to the visible spectrum, other than the increasing values of the absorbance, contributing to the fact that $\mathrm{Ag}$ and Pd exhibit high energy absorbance from other noble metals [12], thus opening up the possibility for new materials and new fields of application.

Author Contributions: Conceptualization, P.P.; methodology, P.P. and M.S.; software, A.G.C. and M.T.; validation, S.G., formal analysis, D.N.; investigation, D.N., D.I.A., A.B., A.G.C. and M.T., resources, P.P. and M.S.; data curation, D.N., M.T. and A.G.C.; writing-original draft preparation, P.P., M.S., M.T. and A.G.C.; writing-review and editing, D.N., A.B., D.I.A. and S.G.; visualization, P.P. and M.S.; supervision, M.S. and P.P.; project administration, S.G.; funding acquisition, D.N., A.B. and A.G.C. All authors have read and agreed to the published version of the manuscript.

Funding: This work was partially financed by the European Union and Greek national funds through the Operational Program Competitiveness, Entrepreneurship and Innovation, under the call RESEARCH-CREATE-INNOVATE (project code: T1EDK-04659).

Institutional Review Board Statement: Not applicable.

Informed Consent Statement: Not applicable.

Data Availability Statement: Data sharing not applicable.

Acknowledgments: V. Karoutsos is acknowledged for the AFM images and N. Bouropoulos for the XRD pattern.

Conflicts of Interest: The authors declare no conflict of interest. 


\section{References}

1. Hutter, E.; Fendler, J.H. Exploitation of localized surface plasmon resonance. Adv. Mater. 2004, 16, 1685-1706. [CrossRef]

2. Haes, A.J.; Hall, W.P.; Chang, L.; Klein, W.L.; Van Duyne, R.P. A localized surface plasmon resonance biosensor: First steps toward an assay for Alzheimer's disease. Nano Lett. 2004, 4, 1029-1034. [CrossRef]

3. Grammatikopoulos, S.; Pappas, S.D.; Dracopoulos, V.; Poulopoulos, P.; Fumagalli, P.; Velgakis, M.J.; Politis, C. Self-assembled au nanoparticles on heated corning glass by dc magnetron sputtering: Size-dependent surface plasmon resonance tuning. $J$. Nanoparticle Res. 2013, 15, 1446. [CrossRef]

4. Guo, F.C.; Sun, T.; Cao, F.; Liu, Q.; Ren, Z. Metallic nanostructures for light trapping in energy-harvesting devices. Light Sci. Appl. 2014, 3, e161.

5. Zhu, W.; Esteban, R.; Borisov, A.G.; Baumberg, J.J.; Nordlander, P.; Lezec, H.J.; Aizpurua, J.; Crozier, K.B. Quantum mechanical effects in plasmonic structures with subnanometre gaps. Nat. Commun. 2016, 7, 11495. [CrossRef] [PubMed]

6. Yang, Q.; Guo, X.; Wang, W.; Zhang, Y.; Xu, S.; Lien, D.H.; Wang, Z.L. Enhancing sensitivity of a single ZnO micro-/nanowire photodetector by piezo-phototronic effect. ACS Nano 2010, 4, 6285-6291. [CrossRef] [PubMed]

7. Pan, C.; Dong, L.; Zhu, G.; Niu, S.; Yu, R.; Yang, Q.; Liu, Y.; Wang, Z.L. High-resolution electroluminescent imaging of pressure distribution using a piezoelectric nanowire LED array. Nat. Photonics 2013, 7, 752-758. [CrossRef]

8. Crossland, E.J.W.; Noel, N.; Sivaram, V.; Leijtens, T.; Alexander-Webber, J.A.; Snaith, H.J. Mesoporous TiO ${ }_{2}$ single crystals delivering enhanced mobility and optoelectronic device performance. Nature 2013, 495, 215-219. [CrossRef] [PubMed]

9. Bernardi, M.; Palummo, M.; Grossman, J.C. Extraordinary Sunlight absorption and one nanometer thick photovoltaics using two-dimensional monolayer materials. Nano Lett. 2013, 13, 3664-3670. [CrossRef] [PubMed]

10. Huang, X.; Tang, S.; Mu, X.; Dai, Y.; Chen, G.; Zhou, Z.; Ruan, F.; Yang, Z.; Zheng, N. Freestanding palladium nanosheets with plasmonic and catalytic properties. Nat. Nanotechnol. 2011, 6, 28-32. [CrossRef]

11. Kumar-Krishnan, S.; Estevez-GonzálezGonz, M.; Esparza, R.; Meyyappan, M. A General seed-mediated approach to the synthesis of $\operatorname{AgM}\left(\mathrm{M} \frac{1}{4} \mathrm{Au}, \mathrm{Pt}\right.$, and Pd) core-shell nanoplates and their SERS properties. RSC Adv. 2017, 7, 27170. [CrossRef]

12. Salem, M.A.; Bakr, E.A.; El-Attar, H.G. Pt@Ag and Pd@Ag core/shell nanoparticles for catalytic degradation of congo red in aqueous solution. Spectrochim. Acta Part. A Mol. Biomol. Spectrosc. 2018, 188, 155-163. [CrossRef]

13. Sui, M.; Kunwar, S.; Pandey, P.; Lee, J. Strongly confined localized surface plasmon resonance (LSPR) bands of Pt, AgPt, AgAuPt nanoparticles. Sci. Rep. 2021, 11, 10319. [CrossRef]

14. Nazemi, M.; Soule, L.; Liu, M.; El-Sayed, M.A. Ambient ammonia electrosynthesis from nitrogen and water by incorporating palladium in bimetallic gold-silver nanocages. J. Electrochem. Soc. 2020, 167, 54511. [CrossRef]

15. Gaylord, T.K.; Moharam, M.G. Rigorous coupled-wave analysis of antireflective surface-relief gratings. J. Opt. Soc. Am. A 1986, 3 , 1780-1787.

16. Lee, M.S.L.; Lalanne, P.; Rodier, J.C.; Chavel, P.; Cambril, E.; Chen, Y. Imaging with blazed-binary diffractive elements. J. Opt. A Pure Appl. Opt. 2002, 4, 358. [CrossRef]

17. Gong, C.; Rebello Sousa Dias, M.; Wessler, G.C.; Taillon, J.A.; Salamanca-Riba, L.G.; Leite, M.S.; Gong, C.; Wessler, G.C.; Taillon, J.A.; Salamanca-Riba, L.G. Near-field optical properties of fully alloyed noble metal nanoparticles. J. Chem. Educ. 2017, 5, 1600568. [CrossRef]

18. Wadell, C.; Yasuhara, A.; Sannomiya, T. Asymmetric light absorption and radiation of Ag-Cu hybrid nanoparticles. J. Phys. Chem. C 2017, 121, 48, 27029-27035. [CrossRef]

19. Nugroho, F.A.A.; Iandolo, B.; Wagner, J.B.; Langhammer, C. Bottom-up nanofabrication of supported noble metal alloy nanoparticle arrays for plasmonics. ACS Nano 2016, 10, 2871-2879. [CrossRef]

20. Lavrenko, V.A.; Malyshevskaya, A.I.; Kuznetsova, L.I.; Litvinenko, V.F.; Pavlikov, V.N. Features of high-temperature oxidation in air of silver and alloy Ag-Cu, and adsorption of oxygen on silver. Powder Metall. Met. Ceram. 2006, 45, 476-480. [CrossRef]

21. Samoylov, A.M.; Ivkov, S.A.; Pelipenko, D.I.; Sharov, M.K.; Tsyganova, V.O.; Agapov, B.L.; Tutov, E.A.; Badica, P. Structural changes in palladium nanofilms during thermal oxidation. Inorg. Mater. 2020, 56, 1020-1026. [CrossRef]

22. Sousanis, A.; Poulopoulos, P.; Karoutsos, V.; Trachylis, D.; Politis, C. Giant enhancement of small photoluminescent signals on glass surfaces covered by self-assembled silver nanorings. J. Nanosci. Nanotechnol. 2017, 17, 1428-1433. [CrossRef] [PubMed]

23. Chronis, A.G.; Stamatelatos, A.; Grammatikopoulos, S.; Sigalas, M.M.; Karoutsos, V.; Maratos, D.M.; Lysandrou, S.P.; Trachylis, D.; Politis, C.; Poulopoulos, P. Microstructure and plasmonic behavior of self-assembled silver nanoparticles and nanorings. J. Appl. Phys. 2019, 125, 23106. [CrossRef]

24. Nilsson, P.O.; Shivaraman, M.S. Optical properties of PdO in the range 05-54 EV. J. Phys. C Solid State Phys. 1979, $12,1423$. [CrossRef]

25. Stamatelatos, A.; Tsarmpopoulou, M.; Chronis, A.G.; Kanistras, N.; Anyfantis, D.I.; Violatzi, E.; Geralis, D.; Sigalas, M.M.; Poulopoulos, P.; Grammatikopoulos, S. Optical interpretation for plasmonic adjustment of nanostructured Ag-NiO thin films. Int. J. Mod. Phys. B 2021, 35, 1-14. [CrossRef]

26. Angelakeris, M.; Poulopoulos, P.; Vouroutzis, N.; Nyvlt, M.; Prosser, V.; Visnovsky, S.; Krishnan, R.; Flevaris, N.K. Structural and spectroscopic magneto-optic studies of Pt-Ni multilayers. J. Appl. Phys. 1997, 82, 5640-5645. [CrossRef]

27. Bukaluk, A.; Rozwadowski, M. Interdiffusion studies in silver/palladium couples by means of auger depth profiling. Vacuum 1995, 46, 579-582. [CrossRef] 
28. Noah, M.A.; Flötotto, D.; Wang, Z.; Mittemeijer, E.J. Interdiffusion and stress development in single-crystalline Pd/Ag bilayers. J. Appl. Phys. 2016, 119, 145308. [CrossRef]

29. Sousanis, A.; Grammatikopoulos, S.; Delimitis, A.; Dracopoulos, V.; Poulopoulos, P. Localized surface plasmon resonances after selective oxidization of AuCu solid solution nanocrystalline films. Appl. Phys. Lett. 2015, 107, 11903. [CrossRef]

30. Karoutsos, V. Scanning probe microscopy: Instrumentation and applications on thin films and magnetic multilayers. J. Nanosci. Nanotechnol. 2009, 9, 6783-6798. [CrossRef]

31. Rakic, A.D.; Djurisic, A.B.; Elazar, J.M.; Majewski, M.L. Optical properties of metallic films for vertical-cavity optoelectronic devices. Appl. Opt. 1998, 37, 5271-5283. [CrossRef] [PubMed]

32. Garoufalis, C.S.; Barnasas, A.; Stamatelatos, A.; Karoutsos, V.; Grammatikopoulos, S.; Poulopoulos, P.; Baskoutas, S. A study of quantum confinement effects in ultrathin $\mathrm{NiO}$ films performed by experiment and theory. Materials 2018, 11, 949. [CrossRef]

33. Barnasas, A.; Kanistras, N.; Ntagkas, A.; Anyfantis, D.I.; Stamatelatos, A.; Kapaklis, V.; Bouropoulos, N.; Mystiridou, E.; Poulopoulos, P.; Garoufalis, C.S.; et al. Quantum confinement effects of thin ZnO films by experiment and theory. Phys. E Low-Dimens. Syst. Nanostructures 2020, 120, 114072. [CrossRef]

34. Fox, M. Optical Properties of Solids, 2nd ed.; Oxford University Press: New York, NY, USA, 2011.

35. Kravets, V.G.; Kabashin, A.V.; Barnes, W.L.; Grigorenko, A.N. Plasmonic surface lattice resonances: A review of properties and applications. Chem. Rev. 2018, 118, 5912-5951. [CrossRef] [PubMed] 